\title{
Variación de color de la serpiente marina Pelamis platura (Serpentes: Elapidae) en el Golfo Dulce, Puntarenas, Costa Rica
}

\author{
Alejandro Solórzano \\ Investigador Asociado, Museo de Zoología, Universidad de Costa Rica, San Pedro de Montes de Oca, San José, Costa Rica \\ Serpentario Nacional de Costa Rica, INBioparque, Santo Domingo, Heredia, Costa Rica; solorzano29@gmail.com
}

Recibido 17-I-2010 Corregido 10-II-2011 Aceptado 28-II-2011

\begin{abstract}
Color variation of the sea snake Pelamis platura (Serpentes: Elapidae) in Golfo Dulce, Puntarenas, Costa Rica. The yellow-bellied sea snake (Pelamis platura) is the most widely distributed of the marine snakes, and throughout its range this species exhibits extensive variation in color and pattern. The coloration of most individuals is black with brown and/or yellow, displayed within a variable pattern, although patternless bright-yellow individuals have been reported from certain localities in Costa Rica and Panama. Recently, within Golfo Dulce in southern Costa Rica, a population was discovered in which all individuals displayed extensive amounts of yellow coloration. The evidence suggests that the circulation patterns of the surface currents within and outside of this fjord-like gulf apparently are strong enough to interrupt the flow (or genetic interchange) among the oceanic and gulf populations. The lack or loss of dark pigment in the dorsum of the Golfo Dulce population might be due to the water temperatures and other specific conditions in the interior of this anoxic basin, the only one in the Pacific coast of Western Hemisphere.
\end{abstract}

\section{KEY WORDS}

Reptilia, Serpentes, Elapidae, Pelamis platura, color pattern, Golfo Dulce, Costa Rica.

\section{RESUMEN}

La serpiente marina de vientre amarillo (Pelamis platura) tiene el mayor ámbito de distribución entre todas las serpientes marinas, y a lo largo de su rango la especie exhibe gran variación en su patrón y color. El color de la mayoría de los ejemplares es negro con café y/o amarillo, que se muestran dentro un patrón variable, aunque ejemplares sin patrón de color amarillo brillante se han reportado de ciertas localidades de Costa Rica y Panamá. Recientemente en el interior de la cuenca del Golfo Dulce, al sur de la costa Pacífica de Costa Rica, se descubrió una población donde todos los ejemplares muestran cantidades extensivas de color amarillo. La evidencia sugiere que los patrones de circulación de las corrientes superficiales en el interior y exterior de este golfo de tipo fiordo, aparentemente son suficientes para interrumpir el flujo o intercambio genético entre la población oceánica y la del Golfo Dulce. La falta o pérdida de la pigmentación oscura en el dorso de la población del Golfo Dulce puede haber sido el resultado de las temperaturas del agua y otras condiciones particulares en esta cuenca anóxica, única en la costa Pacífica del Hemisferio Occidental.

\section{PALABRAS CLAVE}

Reptilia, Serpentes, Elapidae, Pelamis platura, patrón de color, Golfo Dulce, Costa Rica.
La serpiente marina pelágica Pelamis platura es la especie con el mayor ámbito de distribución entre todas las especies de serpientes marinas (Dunson 1975, Kropach 1975, Minton 1975, Greene 1997, Heatwole 1999, Campbell \& Lamar 2004) y es la única especie de serpiente marina presente en la costa oeste de América (Kropach 1975, Tu 1976, Voris 1983, Savage 2002, Savage \& Bolaños 2009, Solórzano 2004, Sasa et al. 2010). En esta región se extiende en aguas tropicales y subtropicales de desde el golfo de California en Norteamérica hasta Ecuador y el norte de Perú en Suramérica, incluyendo las Islas Galápagos y la Isla de Pascua (Campbell \& Lamar 2004). En Costa Rica es común a lo largo de la costa Pacífica (Fig. 1), especialmente en golfos y bahías, por lo general entre 1 y $20 \mathrm{~km}$ de la costa (Solórzano 2004).

A lo largo de su extenso ámbito geográfico exhibe una gran variación en el patrón de color (Fig.2), que se caracteriza por presentar tres tonalidades básicas: negro 


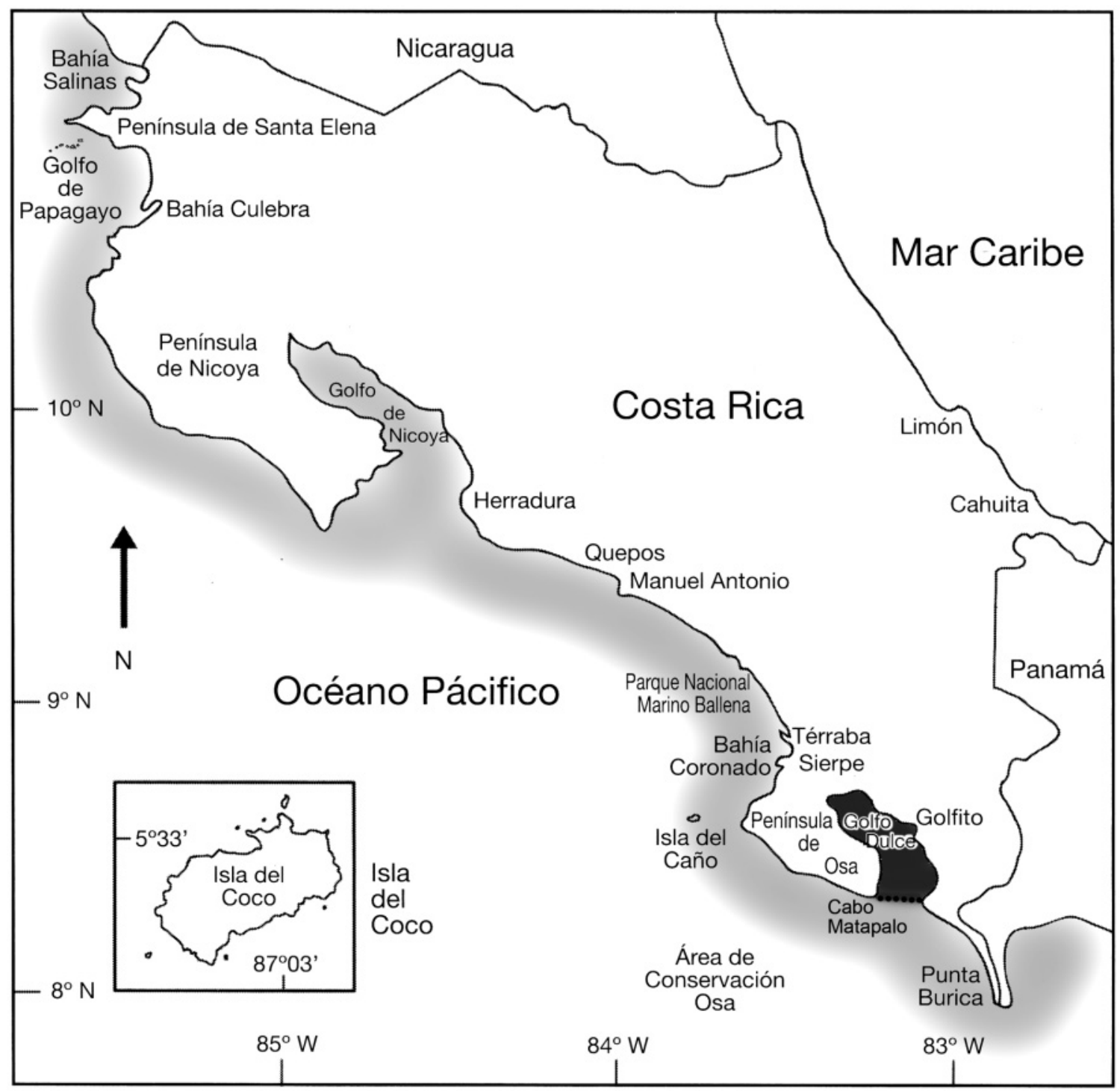

FIG. 1. Distribución de Pelamis platura en la costa Pacífica de Costa Rica. La banda gris clara muestra la población oceánica, y la gris oscura la población interna del Golfo Dulce, posterior al Cabo Matapalo.

uniforme (a veces café o gris oscuro) en el dorso y amarillo y/o café en la región lateral-ventral (Kropach, Tu 1976, Solórzano 2004). En los extremos de esta notable plasticidad fenotípica se observan ejemplares casi totalmente negros y otros completamente amarillos. Los ejemplares con esta vistosa fase de coloración amarilla han sido considerados como muy raros o poco comunes de observar (Kropach 1975, Tu 1976, Voris 1983, Savage 2002, Campbell \& Lamar 2004) y se han reportado únicamente en tres localidades: Bahía Culebra y Golfo Dulce en Costa Rica y en el Golfo de Panamá (Kropach 1975, Tu 1976). Sin embargo, recientemente se comprobó que en el interior del Golfo Dulce, en el sur de la costa Pacífica de Costa Rica, se encuentra una población residente y compuesta en su totalidad por ejemplares completamente amarillos o con solo algunos discretos componentes oscuros sobre el fondo amarillo.

En este trabajo presentamos las características que distinguen a esta población del Golfo Dulce con el resto de las poblaciones oceánicas de esta especie, así como los posibles eventos involucrados en el origen de esta notable variación. 

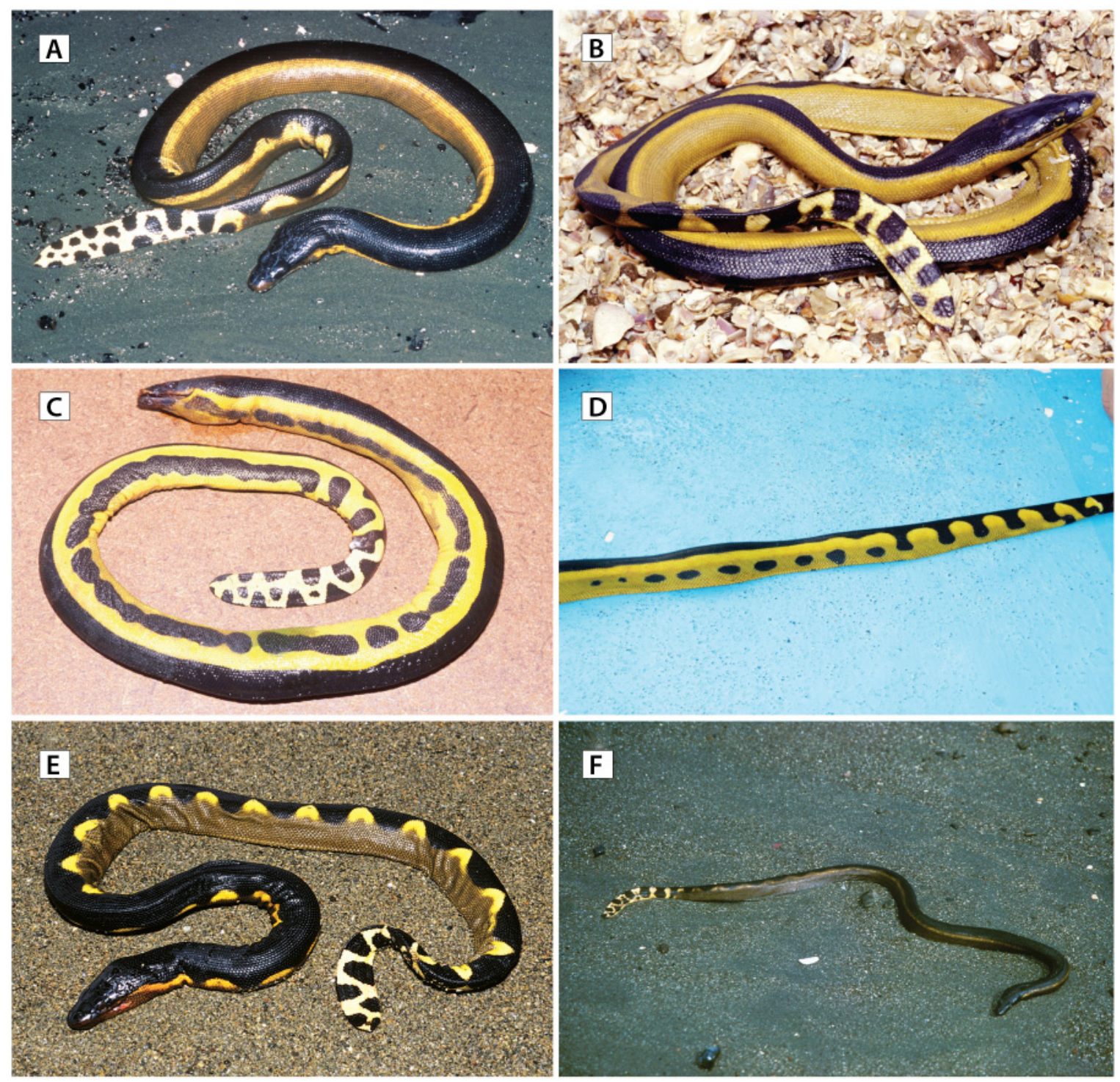

FIG. 2. (A-F) Variación de color en la población oceánica de Pelamis platura en Costa Rica.

\section{METODOLOGÍA}

Este trabajo se realizó entre febrero del 2009 y marzo del 2010. Durante este período se efectuaron 8 viajes de observación y recolecta en el Golfo Dulce, tanto en el interior de la cuenca como en los alrededores de la boca de este golfo. Los recorridos de búsqueda se efectuaron entre la 7:30 y las 11:30 am. En total, se lograron observar 42 serpientes, de las cuales 15 fueron recolectadas para su estudio (Cuadro 1). Tres tipos de medidas se tomaron: longitud hocico-cloaca, longitud total, y longitud de la cabeza. Se registró el estado del clima y la condición de la superficie del mar en el momento en que fueron observados ylo recolectados los ejemplares.

Se realizaron también una serie de entrevistas con pescadores residentes en el área con el fin de obtener una referencia histórica de la presencia y características de estas serpientes.

Se analizaron los patrones de circulación y la temperatura de las corrientes superficiales en el interior y exterior del Golfo Dulce, con base en los estudios de Quesada-Alpízar 
CUADRO 1

Medidas de especímenes de Pelamis platura recolectados en el Golfo Dulce, provincia de Puntarenas

\begin{tabular}{|c|c|c|c|}
\hline Especimen & Longitud hocico-cloaca (mm) & Longitud total (mm) & Longitud cabeza (mm) \\
\hline MZUCR-20612 ㅇ & 541 & 604 & 28 \\
\hline MZUCR-20614 9 & 489 & 545 & 28 \\
\hline MZUCR-20615 $q$ & 555 & 622 & 34 \\
\hline MZUCR-20616 9 & 540 & 598 & 32 \\
\hline MZUCR-20618 + & 502 & 566 & 29 \\
\hline MZUCR-20619 ○َ & 472 & 539 & 27 \\
\hline MZUCR-20648 $q$ & 285 & 320 & 21 \\
\hline MZUCR-20649 ${ }^{\pi}$ & 297 & 336 & 21 \\
\hline MZUCR-20677 $q$ & 443 & 500 & 27 \\
\hline MZUCR-20691 + & 461 & 520 & 29 \\
\hline MZUCR-20817 웅 & 492 & 558 & 30 \\
\hline MZUCR-20818 + & 409 & 564 & 26 \\
\hline MZUCR-20836 ㅇ & 463 & 523 & 29 \\
\hline MZUCR-20837 §̊ & 275 & 305 & 19 \\
\hline MZUCR-20840 ठ̊ & 369 & 419 & 24 \\
\hline
\end{tabular}

\& Morales-Ramírez 2004, Quesada-Alpízar \& Cortés (2006) y Svendsen et al. (2006) y su posible relación con los movimientos de las serpientes marinas entre el sector oceánico periférico a la entrada y en el sector interno de esta cuenca.

\section{Descripción del hábitat}

En Costa Rica P. platura es especialmente abundante en golfos y bahías y por lo general se encuentran asociadas a las líneas o cordones de espuma y residuos orgánicos (Fig. 3) que se forman en la superficie (Kropach 1975, Tu 1976, Solórzano 2004). El Golfo Dulce es un fiordo tropical que se ubica en la parte sur de la costa Pacífica de Costa Rica $\left(8^{\circ} 27^{\prime} \mathrm{N}\right.$ y $\left.8^{\circ} 45^{\prime} \mathrm{N}\right)$. Este ecosistema representa la única cuenca anóxica en la costa Pacífica del Hemisferio Occidental y uno de los cuatro sistemas con estas características que existen en los trópicos (Quesada-Alpízar \& Morales-Ramírez 2004, Quesada-Alpízar \& Cortés 2006, Svendsen et al. 2006). El área posee una superficie aproximada de $680 \mathrm{~km}$ de longitud y de 10 a $15 \mathrm{~km}$ de ancho. Su profundidad máxima alcanza los $215 \mathrm{~m}$, mientras que a su entrada es poco profundo, con alrededor de $60 \mathrm{~m}$, características que tienden a limitar la circulación de agua en la cuenca interna de este golfo. De hecho, la entrada de agua oceánica en la cuenca de este golfo es disminuida en su frecuencia e intensidad debido al umbral externo (Quesada-Alpízar \& Cortés 2006, Svendsen et al. 2006).

\section{RESULTADOS Y DISCUSIÓN}

El patrón de color de los ejemplares de $P$. platura en el Golfo Dulce es amarillo uniforme, ya sea claro o intenso, sin embargo, algunos presentan puntos negros dispersos irregularmente, pequeñas barras longitudinales, líneas continuas o fragmentadas en el centro del dorso, o discretos puntos verdosos y raramente diseños vestigiales en la cola (Fig. 4). Cuando está presente, el diseño más frecuente de observar es una especie de capuchón negro sobre el dorso de la cabeza que cubre las escamas parietales, frontal y supraoculares (Fig. 4 b, c, g).

En el extenso ámbito de distribución de P. platura y su alto grado de variabilidad en el patrón de color, no se habían reportado particularidades específicas a nivel geográfico (Dunson 1975, Kropach 1975, Tu 1976, Campbell \& Lamar 2004, Solórzano 2004). Dentro de este mosaico de variación, los resultados de esta investigación confirman que el patrón amarillo uniforme, que fue tradicionalmente 

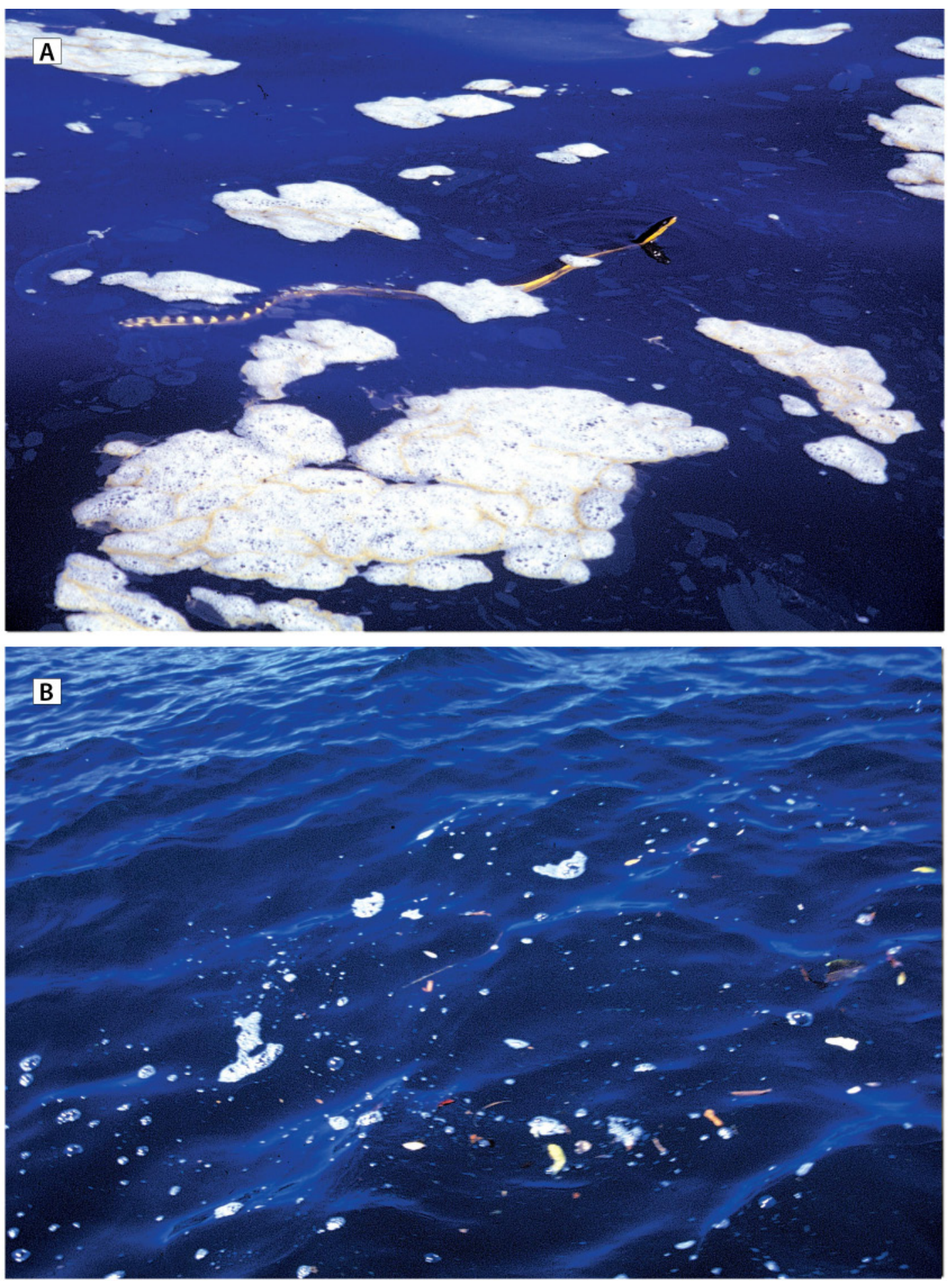

FIG. 3. (A-B) Celdas de convergencia de corrientes o cordones de espuma y desechos orgánicos (también llamadas franjas de flotación o puntos lisos) que se forman en la superficie del mar, y que representan el hábitat más frecuente de Pelamis platura. 

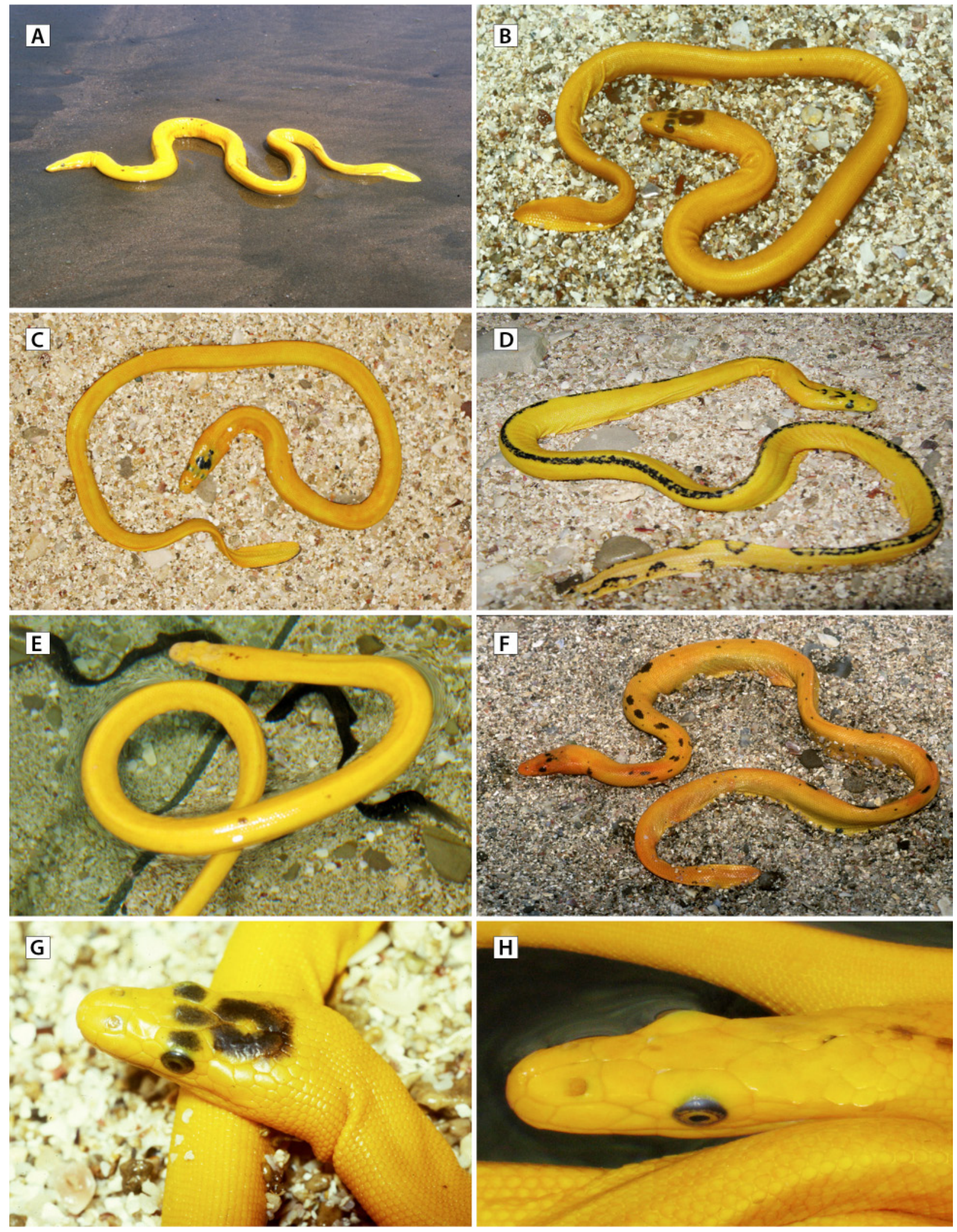

FIG.4. (A-H) Variación de color en la población del Golfo Dulce de Pelamis platura. 
considerado como raro o inusual en la literatura (Kropach 1975, Tu 1976, Voris 1988, Savage 2002, Campbell \& Lamar 2004), no constituye un caso de ejemplares aislados con este fenotipo, sino que se trata de una población residente y claramente establecida con esta característica en el interior de la cuenca del Golfo Dulce. Asimismo, las versiones de varios pescadores consultados y que han sido residentes en el área por períodos que abarcan entre 30 y 40 años, son congruentes con nuestros datos, pues confirman el hecho de que el patrón amarillo uniforme es predominante en las serpientes marinas que se encuentran en el interior del Golfo Dulce y que los ejemplares bicolores o tricolores se localizan hacia el exterior de esta cuenca.

Analizándolo en retrospectiva, es probable que las recolectas reportadas no se realizaran en la parte interna del Golfo Dulce, sino en el sector exterior (conocido como la boca del Golfo Dulce) y posterior al Cabo Matapalo en dirección a mar abierto donde se encuentran las poblaciones oceánicas de patrón bicolor o tricolor de P. platura, como el caso reportado por Voris (1983) donde solamente un tres por ciento de 276 ejemplares recolectados en la "boca del Golfo Dulce" correspondían al patrón amarillo uniforme.

Debido a las características morfológicas particulares de este golfo de tipo fiordo, el flujo e intensidad de agua oceánica que entra hacia el interior de esta cuenca es lento y se ve limitado por el umbral externo de este golfo (Quesada-Alpízar \& Cortés 2006, Svendsen et al. 2006). En la parte interna del golfo, la capa de agua superficial es movida principalmente por los vientos (Svendsen et al. 2006). Es probable que este patrón de circulación constituya una especie de barrera natural que actúe como un elemento potencialmente restrictivo para el tránsito de serpientes marinas oceánicas hacia el interior del Golfo Dulce y viceversa, pues los movimientos de esta especie pelágica están principalmente determinados por las corrientes superficiales (Kropach 1975). Este eventual aislamiento parece interrumpir el flujo reproductivo entre la población de serpientes marinas oceánicas y las del interior del Golfo Dulce, lo que podría favorecer el aislamiento genético que origina este patrón de color, visiblemente dominante en el interior de la cuenca de este golfo. La evidencia sugiere que el sector interior del Golfo Dulce es el centro de origen y dispersión de este notable patrón de color amarillo, por lo que es probable que los escasos ejemplares con este aspecto reportados por Kropach (1975) en el Golfo de Panamá y por Tu (1976) en Bahía Culebra en el noroeste de la costa Pacífica de Costa Rica hayan sido eventualmente trasladados por la dinámica de la Corriente Costera de Costa Rica, que fluye mayormente del sureste al noroeste (Quesada-Alpízar \& Cortés 2006). Un factor que posiblemente esté relacionado con la pérdida del color negro dorsal en esta población es la temperatura del agua superficial, ya que las aguas oceánicas que entran en este golfo se hunden con rapidez, desplazando así las capas internas hacia arriba donde se observan aumentos en la termoclima (Quesada-Alpízar \& Morales-Ramírez 2004, Quesada-Alpízar \& Cortés 2006). Los alcances taxonómicos de esta variación se están estudiando a nivel molecular y se presentarán en la segunda parte de esta investigación (Solórzano et al. en prep.).

Esta especie se encuentra íntimamente asociada a las líneas o cordones de espuma y detritos orgánicos que se forman en la superficie del mar (también llamadas celdas de convergencia) y que representan un hábitat idóneo por la abundancia de recurso alimenticio, así como por las posibilidades de camuflarse entre los escombros flotantes y de apareamiento (Kropach 1975, Solórzano 2004). En el caso del Golfo Dulce, ninguno de los ejemplares observados o colectados se halló dentro de estas líneas superficiales. La mayoría fueron localizados en aguas limpias y tranquilas, con poco viento, lo que parece sugerir que este tipo de hábitat superficial no es determinante para la estabilidad de dicha población.

\section{AGRADECIMIENTOS}

Quiero destacar los valiosos datos, comentarios y sugerencias a lo largo de esta investigación proporcionados por Louis W. Porras, Harvey Lillywhite, Jorge Cortés, Omar Lizano y Héctor Guzmán. De igual manera a Federico Bolaños y Gerardo Chaves por las facilidades brindadas en el Museo Zoología de la Universidad de Costa Rica, así como a Esteban Ocampo por los diseños gráficos y fotográficos. Agradezco también a Catalina Torres (Esquinas Rainforest Lodge) y a Gerardo Sequeira por el apoyo logístico durante el trabajo de campo en el Golfo Dulce, al igual que a Adán Barrera en Playas del Coco y Bahía Culebra, Guanacaste. Este trabajo se realizó bajo el permiso de investigación y colecta del Instituto Costarricense de Pesca y Acuacultura (INCOPESCA) Resolución A. J .D. I. P. 138/2010.

\section{REFERENCIAS}

Campbell, J. A. \& W. W. Lamar. 2004. The venomous reptiles of the Western Hemisphere. 2 vols. Cornell, Ithaca, New York.

Dunson, W. A. 1975. The Biology of the Sea Snakes. University Park, Baltimore MD., 356 p.

Greene, H. W. 1997. Snakes: the evolution of mistery in nature. Univ. California, Berkeley, Los Angeles and London, 351 p.

Heatwole, H. H. 1999. Sea Snakes. 2nd Edition. Krieger Publ., Malabar, Florida, 148 p. 
Kropach, C. 1975. The yellow-bellied sea snake Pelamis platurus in the Eastern Pacific, p. 185-213. In W. A. Dunson (ed.). The Biology of the Sea Snakes. University Park, Baltimore, London and Tokio.

Minton, S. A. 1975. Geographic Distribution of Sea Snakes, p. 21 31. In W. A. Dunson (ed.). The Biology of the Sea snakes. University Park, Baltimore, London and Tokio.

Quesada-Alpízar, M. A. \& A. Morales-Ramírez. 2004. Comportamiento de las masas de agua en el Golfo Dulce, Costa Rica, durante el Niño (1997-1998). Rev. Biol. Trop. 52 (supl.2): 95-103.

Quesada-Alpízar, M. A. \& J. Cortés. 2006. Los ecosistemas marinos del Pacífico sur de Costa Rica: estado del conocimiento y perspectivas de manejo. Rev. Biol.Trop. 54 (Supl. 1): 101-145.

Sasa, M., G. Chaves \& L. W. Porras. 2010. The Costa Rican herpetofauna: Conservation status and future perspectives, P. 510-603. In L.D. Wilson, J. H. Townsend \& J.D. Johnson (eds.). Conservation of Mesoamerican Amphibians and Reptiles. Eagle Mountain Publ, LC., Eagle Mountain, Utah.
Savage, J. M. 2002. Amphibians and Reptiles of Costa Rica: A Herpetofauna between Two Continentes, Two Seas. Univ. Chicago Press, Chicago and London. 934 p.

Savage, J. M. \& F. Bolaños. 2009. A checklist of the amphibians and reptiles of Costa Rica: Additions and nomenclatural revisions. Zootaxa 2005: 1-23.

Svendsen, H., R. Rosland, S. M. Myking, J. A. Vargas, O. G. Lizano \& E. Alfaro. 2006. A physical-oceanographic study of Golfo Dulce, Costa Rica. Rev. Biol. Trop. 54 (Supl. 1): 147-170.

Solórzano, A. 2004. Serpientes de Costa Rica (Snakes of Costa Rica). Instituto Nacional de Biodiversidad (INBio), Santo Domingo, Heredia. 791 p.

Tu, A. T. 1976. Investigation of the Sea Snake Pelamis platurus (Reptilia, Serpentes, Hydrophiidae) on the Pacific coast of Costa Rica, Central America. J. Herpetol. 10: 13-18.

Voris, H. K. 1983. Pelamis platurus, p. 411-12. In D. H. Janzen (ed.). Costa Rican Natural History. Chicago Illinois. 